\title{
ANALISES DE LIVROS
}

ELECTROBIOLOGY OF NERVE, SYNAPSE AND MUSCLE. J. P. REUBEN, D. PURPURA, M. V. L. BENNETT \& E. R. KANDEL, editores. Um volume encadernado (16x24) com 408 págs. Raven Press, New York, 1976. Preço: US $\$ 30,00$.

Este livro foi editado por quatro conhecidos neurofisiologistas em homenagem a Harry Grundfest que, como colaborador de Gasser, na década de 30, foi um dos fundadores da eletrofisiologia moderna. Em seus laboratórios, na Universidade de Columbia (New York) formaram-se numerosos neurofisiologistas que muito contribuiram para a compreensão dos mecanismos fundamentais da gênese, transmissão e processamento de informações no sistema nervoso. Uma das mais peculiares caracteristicas dessa obra conjunta é a abordagem comparativa no estudo dos fenômenos neurais elementares, pela utilização de animais de diversas espécies. Sendo um livro comemorativo o volume contém artigos que tratam de assuntos diversos mas que compartilham de uma linha coerente de investigações científicas; no caso presente essa linha comum é a abordagem eletrofisiológica de fenômenos neurais básicos. $O$ livro consta de 27 artigos divididos em cinco capítulos por afinidades dos assuntos. Alguns dos artigos representam novos enfoques sobre resultados já antes parcialmente publicados por seus autores mas em todos se salienta a excelente qualidade das coñtribuições.

No primeiro capítulo (Excitação axônica), com quatro artigos, deve-se resaltar a curta mais lúcida exposição de Keynes sobre as correntes de comporta que precedem os processos de excitação (e certamente de inibição, embora neste caso ain ja não estudados) da membrana do neurônio, fenômeno para cuja descoberta, feita recentemente, esse fisiologista da Universiuade de Cambridge muito contribuiu. De grande significação, também, é o artigo de Watanabe e Terakawa, em que os autores demonstram a possibilidade de gerar impulsos por aplicação de pulsos de cálcio em solução sem sódio, sem potássio e sem magnésio. O segundo capítulo (Sinapses e interações celulares) constitui-se de dez artigos, dentre os quais se salientam os estudos sobre a transmissão química, sobretudo o de Bennett, Highstein e Model com relação aos fenômenos desenvolvidos na sequestração dos mediadores em vesículas e na reciclagem destas últimas nas terminações sinápticas.

O terceiro capítulo (Integração neural), com seus quatro artigos, apresenta especial interesse para os neurologistas. No primeiro, assinado por Theodore $H$. Bullock, o autor (que é um dos mais respeitados neurofisiologistas da atualidade) faz breve apreciação das propriedades de redundância nos circuitos neurais, defendendo a idéia de que essa proprielade não é tão universal quanto se imagina. Em sua opinião que, aliás, sistematiza a impressão geral que se tem quando se buscam os siñais de redundância no sistema nervoso, "a dificuldade de se provar a completa equivalência e uma real redundância de neurônios contrasta com a relativa facilidade com que se verifica a não-equivalência". O conceito de redundância de circuitos no sistema nervoso era importante para se explicar a compensação cos defeitos decorrentes de lesões nervosas mas a descoberta dos processos de brotamento no SNC permite prescindir-se desse conceito, pelo menos parcialmente, uma vez que pode ser substituído pelo de redundância em série, no tempo, em vez da concomitância em paralelo. Outro artigo importante desse capítulo, é o de Kandel, Carew e Koester, entitulado "Princípios que relacionam as propriedades biofísicas dos neurônios e seus padrões de interconexões com comportamento". Há vários anos Kandel vem estudando sistematicamente os mecanismos de organização de circuitos neurais em organismos menos complexos do que os dos mamíferos, tendo-se fixado na análise desses mecanismos sobretudo em moluscos 
do gênero Aplysia.. Os centros (gânglios) nervosos desses animais têm neurônios em pequeno número e situados em posições relativamente constantes, o que permite sua identificação e até mesmo sua numeração; isso possibilita o estuło de suas conexões e dos mecanismos implicados no funcionamento dos circuitos de que esses neurônios participam. Conseguindo identificar precisamente alguns neurônios ativados (ou desativados) quando comportamentos elementares sãọ desencadeados por estimulação sensorial ou instrumental, os autores sugerem que os padrões comportamentais resultam de interações progressivamente mais complexas de módulos neurais em combinações múltiplas, capazes de gerar os vários comportamentos necessários à viabilidade desses animais. Outro artigo de grande interesse, pela potencialida le das técnicas descritas, é o de S. M. Crain, referente ao desenvolvimento de redes induzidas em cultura de neurônios de gânglios sensitivos. As células que se desenvolvem "in vitro" comportam-se como as homólogas "in vivo", formando redes complexas e emitindo manifestações eletrofisiológicas semelhantes às que ocorrem no organismo intacto.

No quarto capítulo (Acoplamento excitacão-contração e controle de células efetoras), dos oito artigos, cinco tratam dos fenômenos envolvidos na trans lução entre ativação da mebrana e ativação do mecanismo molecular responsável pela contração. Um desses artigos (Papel do cálcio externo no acoplamento excitação-contração em fibras musculares de caranguejo) é de autoria de Suarez-Kurtz e Sorenson, do Departamento de Farmacologia da Universidade Federal do Rio de Janeiro. O último capítulo constitui-se de apenas um artigo, no qual os autores discutem os princípios das técnicas de microiontoforese, que são amplamente utilizadas para introduzir substâncias no interior de neurônios e estudo das consequências dessas microinjeções sobre as características eletrofisiológicas das células. No final descrevem-se as técnicas por eles desenvolvidas no laboratório de Neurofisiologia do próprio homenạgeado e que são atualmente utilizadas rotineiramente nesse laboratório.

Graficamente o volume é bem apresentado; a impressão é boa, as ilustrações muito nítidas e a capa sóbria e agradável. Pela qualidade do conteúdo o livro serve de fonte de informações sobre alguns aspectos fundamentais da Neurofisiologia, ao mesmo tempo em que propicia rara oportunidade para o contacto com pesquisas de vanguarda no campo das neurociências.

CESAR TIMO-IARIA

INFANTILE SPASMS. JOSEPH R. LACY \& J. KIFFIN PENRY. Um volume (16x24) com 169 páginas e 24 tabelas. Raven Press, New York, 1976. Preço: US\$ 15.00.

Espasmos infantis são crises convulsivas próprias da primeira infância, descritas por West em 1841. Frequentemente se acompanham de retardo mental e de uma alteração eletrencefalográfica (hipsarritmia), configurando esta tríade o quałro conhecido como sindrome de West.

O propósito do liyro ora considerado é examinar os aspectos dos espasmos infantis, ressaltando sua importância em Neuropediatria sobretudo devido a três motivos: a resistência à terapêutica antiepiléptica, a incidência elevada de retardo mental e a evolução para outras formas de epilepsia. O material foi disposto em treze capítulos com extensas referências bibliográficas no próprio texto e revisões detalhadas das principais casuísticas encontradas na literatura. Em relação ao quadro clínico os autores descrevem o tipo de crise, discutindo os aspectos considerados essenciais para a caracterização dos espasmos infantis, bem como os elementos variáveis ou modificantes e, finalmente, os fatores precipitantes. Quanto ao retardo mental, que os autores preferem denominar retardo de desenvolvimento em vista c.o grupo etário baixo, são analisadas a época de seu aparecimento, sua concomitância ou não com a fase convulsiva ativa e, com base em estudos de seguimento, a persistência e o grau de retardo mental tardio, após a resolução do quadro clínico e eletrencefálográfico. A 
etiologia é amplamente considerada sendo distinguidos dois grupos: o grupo idiopático, que compreende dois sub-grupos dependendo de haver ou não desenvolvimento normal antes do início dos espasmos, e o grupo sintomático que engloba fatores pré-natais, perinatais e pós-natais. Os achados eletrencefalográficos são descritos não só em relação à hipsarritmia, como também em relação às alterações ictais e a outros tipos de anormalidales não específicas, que eventualmente podem acompanhá-la. A hipsarritmia é analisada mais detalhadamente quanto à sua fisiopatologia, sua relação com a imaturidade cerebral e suas características evolutivas. $\bar{E}$ frisado o fato de a hipsarritmia poder ocorrer em outros tipos de crises, bem como dos espasmos infantis não serem obrigatoriamente ligados à hipsarritmia. O capitulo sobre tratamento é o mais longo, contendo informações valiosas não só quanto à eficácia a curto e a longo prazos das principais drogas no combate às convulsões, como também quanto ao seu possivel efeito sobre o retardo mental e como moditica ioras do quadro eletrencefalográfico. São comparalos os resultados obtidos através da corticoterapia, com os provenientes do uso de benzodiazepínicos, sendo enfatizados aspectos práticos como dosagem, modo e tempo de administração. Os autores analisam ainda outros dados tais como a incilência quanto ao sexo, a possível ocorrência de antecedentes familiares, a eventual concomitância com outros tipos de crises convulsivas e a história natural da moléstia, que compreende a idade, o modo de aparecimento dos espasmos e sua evolução, quanto à frequência, severidade, melhora ou transição para outras formas de epilepsia. Particularmente valiosos são os capítulos sobre taxonomia e anatomia patológica: o primeiro define e especifica os espasmos infantis, individualizando-os como entidade a parte e retiranlo-os tanto da miścelânea de crises mal caracterizadas (crises motoras menores e epilepsia mioclônica da infância), como do grupo das manifestações epilépticas bem definidas clínica e eletrencefalograficamente (sindrome de Lennox-Gastaut e pequeno mal epiléptico); o segundo, apesar das poucas referências bibliográficas a respeito, relata minuciosamente os principais achados de necropsia, diferenciando-os em seis grupos (anormalidades peri- e pós-natais, malformações corticais, desordens metabólicas, alterações degenerativas, miscelânea e sem alterações anátomo-patológicas). Os dados prognósticos são analisados no decorrer da exposição, relacionando-os com a etiologia, a época de aparecimento do retardo mental, a duração do quadro clínico convulsivo, o tipo de crise e a ocorrência de alterações neurológicas. A mortalidade é discutida em capítulo à parte, que compreende uma revisão das diferentes cifras encontradas na literatura, bem como uma análise da possível relação causa-efeito entre os espasmos infantis e o óbito, e da influência da corticoterapia sobre a mortalidade. Finalmente, sob a forma de apêndice é apresentada uma análise comparativa dos principais estudos sobre tratamento já publicados, como tentativa de estabelecer a terapềutica mais eficaz.

Em suma, o conteúdo deste livro praticamente esgota os principais aspectos ligados aos espasmos infantis. Considerando-se além disso que a literatura não é apenas citada mas amplamente revista, sua leitura é obrigatória para o Neuropediatra pelo alto valor informativo, sendo também útil ao Pediatra para alertá-lo sobre a importância do diagnóstico correto e do rápido encaminhamento ao especialista, já que uma razoável porcentagem de casos evolui favoravelmente, quando se institui precocemente a corticoterapia.

UMBERTINA CONTI REED

BIOLOGICAL FOUNDATIONS OF PSYCHIATRY, RCBERT G. GRENELL \& SABIT $G A B A Y$, editores. Dois volumes encadernados $(16 \times 24)$ com 1.044 páginas, 29 tabelas e 126 ilustrações. Raven Press, New York, 1976. Preço: US $\$ 84,00$.

Pode-se definir a Psiquiatria Biológica como o ramo da psiquiatria que se ocupa com: 1) o estudo dos mecanismos centrais que controlam o comportamento; 2) 0 estudo das possibilidades de influenciar estes mecanismos através de meios químicos, elétricos, mecanicos, biológicos, neurofisiológicos, psicofarmacológicos e genéticos; 3 ) o 
desenvolvimento de métodos para o registro adequado do comportamento animal e humano. Esta obra, patrocinada pela Sociedade de Psiquiatria Biológica, é um dos frutos da criação da Federação Mundial de Psiquiatria Biológica durante o primeiro congresso mundial da especialidade, em Setembro de 1974, na cidade de Buenos Aires.

Os dois volumes, a partir da magnífica introdução por Sir John Carew Eccles, apresentam cs seguintes capítulos: 1 - A genética no comportamento e na Psiquiatria (John D. Rainer); 2 - O processo sensório, percepcão e comportamento (Robert B. Livingston); 3 - Especializaç̃o hemisférica: implicasões para a Psiquiatria (David Galin); 4 - Fatores sensórios e perceptuais nas funções do "cérebro triuno (Paul D. MacLean); 5 - Potenciais evocados no homem (Charles Shagass); 6 - Emocão $e$ percepcão sensorial: estudos animais $e$ humanos (Robert G. Heath); 7 - Fundamentos neurais da ameaça $e$ do ataque (John P. Flynn); 8 - Fome $e$ sede (J. A. Deutsch e H. S. Koopsmans); 9 - Estudos comportamentais das funções hipotalâmicas: impulsos e reforços (James Olds); 10 - Progressos recentes na psicofisiologia do sono e o seu significado psiquiátrico (Ismet Karacan, A. Michael Anoh e Robert L. Williams); 11 - Aspectos neurobiológicos da memória (James L. McGaugh); 12 - A análise neuropsicológica do deficit cognitivo (M. Kinsbourne); 13 - Neurobiologia do desenvolvimento (Williamina A. Himwich); 14 - Fatores genéticos na esquizofrenia (Ming $T$. Tsuang); 15 - Genética de distúrbio afetivo (Remi J. Cadoret); 16 - A bioquimica da esquizofrenia (Ronald J. Bradley e John R. Smythies); $17-$ A bioquimica dos distúrbios afetivos (H. Weil-Malherbe); 18 - Alucinacões quimicas e fisiológicas (Arnold J. Mandell e Mark A. Geyer); 19 - Psicoses modelo (Charles E. Frohman e Jacques S. Gottlieb); 20 - Psicoquimioterapia (Arthur K. Shapiro); 21 - Cinética das drogas antipsicóticas e antidepressivas no homem (Leo E. Hollister); 22 - Tolerâancia e dependência a drogas (Seymour Ehrenpreis, Davia N. Teller e Abel Lajtha); 23 - Stress, ansiedade, e função endócrina (Arthur Yuwiler); 24 - Correlatos biológicos de doença psicossomática (James P. Henry e Daniel L. Ely); 25 - Um modelo processador de informaç̃es da mente (R. G. Grenell e D. McCulloch); 26 - Neuropsiquiatria e neuropsicologia (Karl H. Pribram). O primeiro volume aboria mais aspectos neurobiológicos enquanto que, no segundo, predominam os aspectos bioquímicos e farmacológicos relacionados às doenças mentais.

Ao contrário do que o título possa sugerir, este não é um trata ło de psiquatria biológica, mas tão-somente uma coletânea de revisões e trabalhos originais realizados por algumas das maiores autoridades no assunto. Como tal, não existe uma uniformidade editorial, constatando-se também a existência de algumas omissões, o que aliás é reconhecido pelos próprios editores no prefácio. Conquanto sejam altamente recomendáveis aos iniciados, estes dois volumes são também indicados aos que desejam adquirir um consenso, e quiçá alguns fundamentos, sobre a Psiquiatria Biológica.

MAURO SALOMÃO WEINTRAUB

EPILEPSY. A CLINICAL, ELECTROENCEPHALOGRAPHIC AND STATISTICAL STUDY OF 466 PATIENTS. TAKAYUKI TSUBOI \& WALTER CHRISTIAN. Um volume $(17 \times 24,5)$ encadernado com 107 páginas, 11 figuras e 45 tabelas. Volume 17 da série Schriftenreihe Neurologie (texto em inglês). Springer Verlag, BerlinHendelberg-New York, 1976. Preço: US\$ 24,00.

As epilepsias têm sido muito estudadas nestes últimos 20 anos mediante abordagens diversificadas dos elementos clínicos e dos achados eletrencefalográficos. Numerosos trabalhos, além de estudos de aspectos particulares às epilepsias, cuidaram de correlacionar fatores diversos que podem alterar a sintomatologia clínica e as anormalidades eletrencefalográficas produzindo síndromes peculiares à infância, ao estado jovem, à 
adultidade e à velhice. Entretanto, no livro que está sendo comentado os autores tiveram a paciência de coletar dados relativos a 466 doentes registrałos no Departamento de Neurologia da Faculdade de Melicina da Universidade de Heidelberg e fazer uma correlação sistemática e multidisciplinar dos daরos obtidos, comparando seus resultados com aqueles fornecidos pela literatura pertinente. Foram revisadas as observações de 500 pacientes consecutivos (de um total de 7.400 pacientes registrados), sendo eliminados 34 cujas observações não continham material suficiente para análise estatística precisa. Foram estudados a idade e o sexo dos pacientes, os tipos de crises, as datas do início das crises, as anormalidades eletrencefalográficas paroxísticas, o rítimo eletrencefalográfico de base, a predisposição ramiliar e a influência dos fatores exógenos. A análise estatística foi feita mediante aplicação do teste X2, do teste exato de Fischer e do teste de Student, sendo feita também a análise do coeficiente de contingência. Todos os dados foram correlacionados entre sí, sendo os resultados apresentados nas $\mathbf{4 5}$ tabelas que enriquecem este pequeno mas valioso livro cuja leitura permitirá aos epileptólogos comparar seus dados pessoais com aqueles registrados na literatura e concernentes aos dados particulares estudados por diversos autores e, especialmente, compará-los com os dados globais coletados agora por Takayuki Tsuboi e Walter Christian que realizaram trabalho de grande importância e subido valor. Só a leitura do livro permitirá avaliar o vulto do material de estudo nele coletado.

\section{O. LANGE}

GLOSSAIRE ET GUIDE DE CLASSIFICATION DES TROUBLES MENTAUX. Vários autores. Opúsculo com 91 páginas editado pela Organização Mundial da Saúde, Genebra, 1976. Publicado em francês, inglês e espanhol. À venda na Biblioteca Regional de Medicina OMS/OPS, Caixa Postal 20381, Vila Clementino, 01000 São Paulo SP, Brasil. Preço: $\mathrm{Cr} \$ 65,00$.

Este glossário deve ser usado em ligação com a Classificação Internacional de Doenças, $8^{a}$ revisão (CIM-8) no capítulo relativo à Psiquiatria, tendo sido preparado por um grupo de trabalho designado especialmente pela OMS que manteve contactos com psiquiatras e peritos de mais de 60 países. O glossário contém descrições concordantes no plano internacional para cada uma das rúbricas de diagnóstico, sendo descritos os sintomas e outros característicos dos estados mórbidos. Nos casos em que ocorreram diferenças de uso nos diferentes países, o glossário indica igualmente os sintomas e síndromes que devem ser excluídos das codificações internacionais. Muitas rúbricas são seguidas de sinónimos aceitáveis. A fim de permitir pesquisas rápidas e facilitar a codificação ajustała de qualquer diagnóstico, todas as denominações, incluindo sinonimia, foram ajuntadas em índice alfabético. $O$ opúsculo contém também um certo número de seções que fornecem informações complementares para a classificação. A introdução contém generalidades sobre o problema da classificação das doenças em geral e, em particular, das doenças mentais. O modus faciendi para o emprego do glossário é bem formulado, mostrando como se pode utilizá-lo, sendo propostos diversos sistemas de codificação nos quais pode ser aplicado mais de um diagnóstico. Os anexos 1 e 2 constituem, respectivamente, uma apresentação e um esquema da CIM-8 e têm por objetivo mostrar às pessoas que utilizam o capítulo V da CIM (distúrbios psíquicos), como é possível registrar estados mórbidos associados. Para facilitar o trabalho dos diagnosticadores e dos codificadores, o anexo 3 fornece uma lista dos estados mórbidos que frequentemente conduzem a contradições diagnósticas entre os diversos serviços psiquiátricos de diferentes países. 
CLASSIFICATION MULTI_AXIALE DES TROUBLES PSYCHIATRIQUES DE L'ENFANT. M. RUTTER, D. SHAFFER \& M. SHEPPERD. Opúsculo com 96 páginas, editado pela Organização Mundial de Saúde, 1975. Publicado em francês e inglês. À venda na Biblioteca Regional de Medicina OMS/OPS, Caixa Postal 20381, Vila Clementino, 01000 São Paulo SP, Brasil. Preço: Cr\$ 90,00.

Esta classificação multi-axial dos distúrbios psiquiátricos da criança relata, inicialmente, os trabalhos para a busca de um sistema enquadrável na Classificação Internacional de Doenças, levando em conta a fidelidade, a validez e a utilização prática dos dados nela contidos. A classificação comporta quatro eixos, cada um deles constituído por uma lista de rúbricas e códigos, como sejam: o síndromo psiquiátrico infantil, o nivel intelectual, os fatores biológicos, as influências psíco-sociais associadas ou etiológicas. Antes de iniciar o trabalho encomendado pela OMS os participantes (22 psiquiatras ingleses) se reuniram por diversas vezes para assegurar a uniformidade técnica dos exames a serem feitos nos pacientes, para considerar os objetivos visados e para uniformizar a metódica a ser empregada. A classificação resultante de todo este trabalho apresenta importantes vantagens sobre as classificações atualmente utilizadas e mesmo sobre a CIM-8, especialmente quando um distúrbio psiquiátrico se associa a uma afecção somática ou a um retardo intelectual. Ela fornece informações mais sistematizadas e sob uma forma mais comparável, permitindo distinguir mais claramente o sin .rome clínico psiquiátrico dos fatores etiológicos que o influenciam. Além disso é de aplicação mais cômoda porque corresponde mais estritamente à abordagem clínica habitual, sendo, portanto, mais útil na prática corrente. A codificação dos síndromes psiquiátricos utilizada nesta classificação teve alto índice de fidelidade na grande maioria dos casos estudados; apesar disso foi recomendado que fossem feitas novas pesquisas para ajustamento da abordagem relativa às influências psíco-sociais. Com essa ressalva, foi dado um passo decisivo para a elaboração futura de uma classificação racional dos distúrbios psiquiátricos que ocorrem em crianças.

Comunicado da OMS 